\title{
Combining Ability for Yield and Its Attributing Traits in Okra [Abelmoschus esculentus (L.) Moench]
}

\author{
K. Satish ${ }^{1 *}$, A.V. Agalodiya ${ }^{2}$ and D.B. Prajapati ${ }^{2}$ \\ ${ }^{1}$ Department of Genetics and Plant Breeding, S.D. Agricultural University, \\ Sardarkrushinagar, Gujarat - 385 506, India \\ ${ }^{2}$ Seed Spices Research Station, S.D. Agriculture University, Jagudan, Gujarat - 382 710, India \\ *Corresponding author
}

\begin{tabular}{|c|c|}
\hline & A B S T R A C T \\
\hline Keywords & \multirow{6}{*}{$\begin{array}{l}\text { The experiment was undertaken to study the combining ability for yield and its attributing } \\
\text { traits in okra. Information on the magnitude of combining ability was obtained for fruit } \\
\text { yield per plant and its related components following line x tester mating design involving } \\
15 \text { diverse varieties/strains ( } 10 \text { lines and } 5 \text { testers). The } 15 \text { parents and their } 50 \text { resultant } \\
\text { F }_{1} \text { s were sown in a Randomized Block Design with three replications. Combining ability } \\
\text { analysis revealed that the magnitude of non-additive variance was higher for fruit yield per } \\
\text { plant and its contributing traits indicating the predominant role of non-additive gene action } \\
\text { in the inheritance of the traits. Among females, JOL-08-7 (24.60) while, among males, } \\
\text { Parbhani Kranti ( } 8.11 \text { ) were good general combiners for fruit yield per plant and related } \\
\text { traits. The hybrid, JOL- } 08-7 \mathrm{x} \text { Parbhani Kranti ( } 37.08) \text { having high sca effects for fruit } \\
\text { yield per plant also registered desirable } s c a \text { effects for a number of branches per plant, } \\
\text { internodal length, fruit girth and days to } 50 \text { per cent flowering. }\end{array}$} \\
\hline & \\
\hline $\begin{array}{l}\text { Line } x \text { Tester, } \\
\text { Combining ability }\end{array}$ & \\
\hline Fruit yield, Okra. & \\
\hline Article Info & \\
\hline $\begin{array}{l}\text { Accepted: } \\
\text { 21 July } 2017 \\
\text { Available Online: } \\
\text { 10 September } 2017\end{array}$ & \\
\hline
\end{tabular}

\section{Introduction}

Okra (Abelmoschus esculentus (L.)Moench) belonging to the family Malvaceae is an important vegetable crop of the tropics and subtropics. Okra is specially valued for its tender, delicious green fruits which are cooked, canned and consumed in various forms in different parts of the country. Major areas of cultivation in India are Uttar Pradesh, Bihar, Orissa, West Bengal, Andhra Pradesh, Gujarat, Karnataka and Assam. Okra is a polyploid, belonging to the family Malvaceae with $2 \mathrm{n}=8 \mathrm{x}=72$ or 144 chromosomes and a self-pollinated crop, the occurrence of out crossing to an extent of $4-19 \%$ with the maximum of $42.2 \%$ is noticed with the insect assisted pollination (Kumar, 2006). India is a major okra producing country in the world comprising of $72 \%$ of total area under okra (FAOSTAT, 2013). The area under okra cultivation in India is 503.68 ('000 ha) with the production of 5708.69 ('000 mt) and productivity of $11.5 \mathrm{mt} / \mathrm{ha}$, containing 5.7 per cent of the total area under vegetable crops and 3.9 per cent of total vegetable production (Anonymous, 2015). It is also an important vegetable crop in Gujarat state and is mainly grown in Banaskantha, Bhavnagar, Junagadh, 
Navsari, Surat, Tapi and Vadodara Districts. It occupies an area of 73.84 ('000 ha) with a production of 857.49 ('000 $\mathrm{mt}$ ) having the average productivity of $11.5 \mathrm{mt} / \mathrm{ha}$. West Bengal is a leading okra producing state with 14 per cent of production share, while Gujarat comprised of 12 per cent production share (Anonymous, 2015). The knowledge of nature of gene action governing the expression of various traits could be helpful in predicting the effectiveness of selection. The combining ability is the important genetic tool, which provides a guideline for an assessment of the relative breeding potential of the parents or identifying the best combiners, which may be hybridized either to exploit heterosis or to accumulate fixable genes (Singh et al., 1991). Several approaches are available for assessing the parents and cross combinations with respect to combining ability. Among these, Line $\mathrm{x}$ Tester analysis technique provides a systematic approach for identification of superior parents and crosses, which is the basic requirement on which the success of a breeding programme rests (Singh et al., 2006 and Khatik et al., 2012). In any sound breeding programmes, the proper choice of parents based on their combining ability is a prerequisite. The studies intended to determine the combining ability is not only for information regarding the choice of parents but also for the production of superior lines or hybrids. The general combining ability and specific combining ability effects are the foundations for any fruitful breeding programme (Wakode et al., 2016). The common approach of selecting the parents on the basis of per se performance is not a good indicator of their superior combining ability (Allard, 1960).

\section{Materials and Methods}

The experimental material consisted 10 female parents (lines) viz., JDNOL-11-1 (L1), JDNOL-11-3 (L2), JDNOL-11-11 (L3),
JDNOL-11-12 (L4), JDNOL-11-14 (L5), AOL-07-9 (L6), AOL-08-5 (L7), JOL-6k-2 (L8), JOL-08-7 (L9), JOL-08-12 (L10) and 5 male parents (testers) namely, Arka Anamika (T1), Pusa Sawani (T2), Arka Anamika (T3), GO-2 (T4) and VRO-6 (T5). These parental lines were selected on the basis of per se performance, adoption and geographical diversity and crossed in the line $\mathrm{x}$ tester design for producing 50 hybrids. All the 50 $\mathrm{F}_{1} \mathrm{~s}$ and their 15 parents alone with one check (GJOH-3) were grown in randomised block design with three replications at Seed Spices Research Station, S. D. Agricultural Universtiy, Jagudan in kharif 2014. The seeds were sown in a single row of $3.6 \mathrm{~m}$ length keeping $60 \mathrm{~cm}$ distance between row and 45 $\mathrm{cm}$ within the row for easy movement. All packages of cultivation practices were followed to raise a healthy crop. The observations were recorded on all the five plant chosen at random in each genotype and in each replication and following observations were recorded for yield and its contributing traits viz., days to 50 per cent flowering, days to first picking, fruit length $(\mathrm{cm})$, fruit girth (cm), number of branches per plant, internodal length $(\mathrm{cm})$, plant height $(\mathrm{cm})$, total number of fruits per plant, fruit yield per plant (g), total number of seeds per fruit and days to last picking. Data was analyzed to work out the combining ability of the parents as well as the crosses (Kempthrone, 1957).

\section{Results and Discussion}

In the present study, line $\mathrm{x}$ tester method was applied to develop 50 experimental hybrids from 10 lines (females) and 5 testers (males), which were evaluated under kharif season. Analysis of variance (mean squares) and variance estimates for combining ability for different traits are presented in table 1. A perusal of variance components revealed that the mean squares due to crosses were highly significant for all the traits except days to last 
picking. A perusal of variance components revealed that the mean squares due to lines and testers were non-significant for all the characters except fruit yield per plant in lines.

The mean squares due to line $\mathrm{x}$ tester were significant for all the characters except number of seeds per fruit and days to the last picking signifying that both females and males had considerable general combining ability $(\mathrm{gca})$ towards these traits and contributed towards additive genetic variance. Highly significant mean squares due to females $x$ males were manifested by all the traits reflecting its significant contribution in favour of specific combining ability (sca) and non-additive variances. An assessment of gcaof females ( $\sigma^{2}$ females) and gca of males (males) for their contribution towards gca (average $g c a$ ) revealed that for all the traits females contributed largely towards $g c a$, emphasizing the importance of additive and non-additive gene actions in the inheritance of these traits. This is expected since the divergent material was included in the study. Same results were obtained by Sood and Kalia (2001). The additive and non-additive gene effects could be exploited in okra by the use of good general combiners in hybridization and selecting desirable segregants from segregating generations.

However, a perusal of $\mathrm{gca} / \mathrm{sca}$ ratio revealed a preponderance of non-additive gene action for all the traits. As preponderance of nonadditive gene action and high heterosis is apparent for fruit yield and majority of yield contributing traits, it is recommended that heterosis breeding could be used for exploitation of hybrid vigour on a commercial scale. The importance of non-additive genetic variances for fruit yield per plant has been reported by several workers such Jayprakashnarayan et al., (2008), Singh et al., (2009), Wammanda et al., (2010), Khatik et al., (2012), Medagam et al., (2012), Obiadalla
Ali et al., (2013), Jethava (2014) and Katagi et al., (2015).

The character wise estimates of general combining ability effects for each parent are presented in table 2 and indicates that the merit of the parents differs significantly for different characters. Analysis of data revealed that among parents, line AOL-08-5 (-1.69) and JOL-08-12 (1.08) showed significant negative $\mathrm{gca}$ effect for days to 50 per cent flowering which is desirable and contribute for earliness in crosses. Whereas none of the testers found with negative significant gca effect. In the case of number of seeds per fruit desired highest gca effects in the desired direction (significant negative) were exhibited by line JDNOL-11-1 (-1.86). In case of a number of branches per plant and fruit yield per plant desired highest gca effects in the desired direction (significant positive) were exhibited by line JOL-08-7 and JOL-08-12 are good combiners for these traits. As far as fruit length and fruit girth are yields attributing traits, female parent, JDNOL-1114 (0.54) exhibited higher gca effects for fruit length. Among parents, female parent, JDNOL-11-3 (0.44) exhibited significant gca effects for the trait fruit girth whereas, none of the male parents exhibited significant $g c a$ effects for traits fruit length and fruit girth.

In case of days to first picking and days to last picking line, JOL-08-7 (-2.72) showed significant gca effects for days to first picking while none of the line or tester exhibit significant gca effect in the desired direction. Among parents line, AOL-07-9 (-9.00) and tester, GO-2 (-6.29) for plant height and line JDNOL-11-11 (1.14) exhibited significant gca effect in desired direction for traits plant height and number of fruits per plant. Among all 15 parents, line, JOL-08-7 and JOL-08-12 proved to be the best general combiner for yield and yield attributing characters. 
Int.J.Curr.Microbiol.App.Sci (2017) 6(9): 1944-1954

Table.1 Analysis of variance (mean squares) and variance estimates for combining ability for different trait

\begin{tabular}{|c|c|c|c|c|c|c|c|c|c|c|c|c|}
\hline \multicolumn{13}{|c|}{ Traits } \\
\hline Sources & d.f. & $\begin{array}{c}\text { Days to } \\
50 \% \\
\text { flowering }\end{array}$ & $\begin{array}{c}\text { Days to } \\
\text { first } \\
\text { picking }\end{array}$ & $\begin{array}{l}\text { Fruit } \\
\text { length } \\
(\mathrm{cm})\end{array}$ & $\begin{array}{l}\text { Fruit } \\
\text { girth } \\
(\mathrm{cm})\end{array}$ & $\begin{array}{c}\text { Number } \\
\text { of } \\
\text { branches } \\
\text { per plant }\end{array}$ & $\begin{array}{c}\text { Internodal } \\
\text { length } \\
\text { (cm) }\end{array}$ & $\begin{array}{c}\text { Plant } \\
\text { height } \\
(\mathrm{cm})\end{array}$ & $\begin{array}{c}\text { Fruit } \\
\text { yield per } \\
\text { plant (g) }\end{array}$ & $\begin{array}{c}\text { Number } \\
\text { of fruits } \\
\text { per } \\
\text { plant }\end{array}$ & $\begin{array}{c}\text { Number } \\
\text { of seed } \\
\text { per fruit }\end{array}$ & $\begin{array}{c}\text { Days to } \\
\text { last } \\
\text { picking }\end{array}$ \\
\hline Replication & 2 & 6.22 & 8.37 & 0.14 & 0.15 & 0.04 & 0.91 & $852.38 * *$ & $1405.00 *$ & 0.16 & 1.08 & 9.89 \\
\hline Hybrids & 49 & $15.70 * *$ & $23.35 * *$ & $1.07 * *$ & $1.59 * *$ & $0.21 * *$ & $3.05^{* *}$ & $685.16 * *$ & $1916.34 * *$ & $10.79 * *$ & $14.95^{*}$ & 59.92 \\
\hline Line $(F)$ & 9 & 13.21 & 32.34 & 1.42 & 1.28 & 0.25 & 4.97 & 641.07 & $3667.24 *$ & 7.62 & 22.27 & 96.22 \\
\hline Tester (M) & 4 & 34.45 & 20.19 & 1.92 & 1.32 & 0.21 & 1.44 & 860.04 & 902.07 & 7.12 & 15.94 & 33.53 \\
\hline Line $\mathrm{x}$ Tester & 36 & $14.24 * *$ & $21.45 * *$ & $0.89 * *$ & $1.70 * *$ & $0.19 * *$ & $2.75^{* *}$ & $676.76 * *$ & $1591.32 * *$ & $11.99 * *$ & 13.01 & 53.78 \\
\hline Error & 98 & 4.68 & 8.80 & 0.31 & 0.15 & 0.03 & 1.19 & 58.66 & 382.70 & 2.99 & 9.35 & 41.42 \\
\hline \multicolumn{13}{|l|}{ Estimates } \\
\hline$\sigma^{2}$ Females & - & 0.58 & 1.62 & 0.07 & 0.07 & 0.01 & 0.26 & 35.07 & $214.47 *$ & 0.31 & 0.87 & 3.37 \\
\hline$\sigma^{2}$ Males & - & 1.00 & 0.40 & 0.05 & 0.03 & 0.006 & 0.01 & 24.83 & 15.06 & 0.14 & 0.22 & -0.40 \\
\hline$\sigma^{2}$ gca & - & $0.86 * *$ & $0.81 *$ & $0.06^{* *}$ & 0.05 & $0.009 *$ & $0.09 *$ & $28.25^{*}$ & $81.53 * *$ & 0.20 & $0.43 * *$ & $0.85 *$ \\
\hline$\sigma^{2}$ sca & - & $3.26 * *$ & $4.49 * *$ & $0.19 * *$ & $0.51 * *$ & $0.05^{* *}$ & $0.59 * *$ & $187.29 * *$ & $80.37 * *$ & $3.04 * *$ & 1.26 & 2.74 \\
\hline$\sigma^{2} \mathrm{gca} / \sigma^{2} \mathrm{sca}$ & - & 0.26 & 0.18 & 0.31 & 0.09 & 0.16 & 0.16 & 0.15 & 0.21 & 0.06 & 0.34 & 0.31 \\
\hline
\end{tabular}

$*$ And ** significant at $5 \%$ and $1 \%$ level of probability, respectively. 
Table.2 Estimates of general combining ability (gca) effects for different traits in okra

\begin{tabular}{|c|c|c|c|c|c|c|c|c|c|c|c|}
\hline \multicolumn{12}{|c|}{ Traits } \\
\hline Genotypes & $\begin{array}{c}\text { Days to } \\
50 \% \\
\text { flowering }\end{array}$ & $\begin{array}{l}\text { Days to } \\
\text { first } \\
\text { picking }\end{array}$ & $\begin{array}{l}\text { Fruit } \\
\text { length } \\
(\mathrm{cm})\end{array}$ & $\begin{array}{l}\text { Fruit } \\
\text { girth } \\
(\mathrm{cm})\end{array}$ & $\begin{array}{l}\begin{array}{c}\text { Number } \\
\text { of } \\
\text { branches } \\
\text { per plant }\end{array}\end{array}$ & $\begin{array}{l}\text { Internodal } \\
\text { length } \\
(\mathrm{cm})\end{array}$ & $\begin{array}{l}\text { Plant } \\
\text { height } \\
\text { (cm) }\end{array}$ & $\begin{array}{l}\text { Fruit } \\
\text { yield } \\
\text { per } \\
\text { plant } \\
\text { (g) }\end{array}$ & $\begin{array}{c}\text { Number } \\
\text { of fruits } \\
\text { per plant }\end{array}$ & $\begin{array}{l}\text { Number } \\
\text { of seed } \\
\text { per fruit }\end{array}$ & $\begin{array}{c}\text { Days to } \\
\text { last } \\
\text { picking }\end{array}$ \\
\hline \multicolumn{12}{|l|}{ Lines } \\
\hline JDNOL-11-1 & 0.69 & 0.47 & -0.05 & -0.09 & $-0.15 * *$ & 0.04 & $-7.02 *$ & $-24.95 * *$ & 0.13 & $-1.86^{*}$ & -2.97 \\
\hline JDNOL-11-3 & -0.61 & $1.49 *$ & -0.01 & $0.44 * *$ & 0.04 & $-0.56 *$ & $-6.59 *$ & 0.70 & 0.28 & -0.67 & -0.04 \\
\hline JDNOL-11-11 & 0.10 & -0.40 & 0.19 & -0.09 & $-0.09 *$ & $0.81 * *$ & $7.62 * *$ & $-12.25^{*}$ & $1.14 *$ & 0.40 & 2.38 \\
\hline JDNOL-11-12 & 0.71 & 1.25 & -0.16 & -0.05 & -0.06 & $-0.51 *$ & -0.006 & -0.02 & -0.39 & -0.48 & 1.45 \\
\hline JDNOL-11-14 & -0.46 & $-1.74 *$ & $0.54 * *$ & 0.18 & $0.17 * *$ & $0.78 * *$ & 1.57 & 4.76 & 0.47 & 0.44 & 1.72 \\
\hline AOL-07-9 & 0.71 & $1.44 *$ & -0.03 & $-0.55^{* *}$ & $-0.20 * *$ & -0.36 & $-9.00 * *$ & -6.03 & -0.35 & -1.10 & $-4.88 * *$ \\
\hline AOL-08-5 & $-1.69 * *$ & -0.45 & 0.17 & $0.32 * *$ & 0.04 & 0.04 & $7.55 * *$ & $12.59 *$ & -0.19 & $1.90 *$ & 2.85 \\
\hline JOL-6k-2 & $1.29 *$ & 1.38 & $-0.52 * *$ & $-0.27 *$ & -0.02 & $-0.86 * *$ & -3.65 & $-17.07 * *$ & $-1.55^{* *}$ & -0.84 & -2.14 \\
\hline JOL-08-7 & 0.31 & $-2.72 * *$ & 0.24 & 0.15 & $0.13 * *$ & 0.15 & 0.62 & $24.60 * *$ & 0.45 & $1.73^{*}$ & 1.10 \\
\hline JOL-08-12 & $-1.08 *$ & -0.73 & $-0.36^{*}$ & -0.03 & $0.14 * *$ & 0.45 & $8.90 * *$ & $17.66 * *$ & 0.002 & 0.48 & 0.51 \\
\hline S.E.g ${ }_{i}$ & 1.08 & 1.44 & 0.28 & 0.20 & 0.08 & 0.50 & 5.49 & 10.87 & 0.86 & 1.55 & 3.45 \\
\hline \multicolumn{12}{|l|}{ Testers } \\
\hline ArkaAnamika & $1.87 * *$ & $1.28 *$ & $-0.26^{*}$ & $-0.22 * *$ & $-0.12 * *$ & -0.02 & 0.59 & -6.15 & -0.49 & -0.35 & 0.25 \\
\hline PusaSawani & -0.63 & -0.57 & 0.18 & $0.17 *$ & 0.01 & 0.26 & -2.22 & -0.95 & 0.38 & -0.19 & 1.36 \\
\hline ParbhaniKranti & -0.75 & -0.76 & 0.09 & $0.21 * *$ & $0.07 *$ & -0.18 & $8.27 * *$ & $8.11 *$ & 0.09 & 0.60 & -1.43 \\
\hline GO-2 & -0.31 & 0.28 & $-0.27 * *$ & $-0.21 * *$ & -0.04 & -0.23 & $-6.39 * *$ & 2.24 & -0.51 & -0.92 & -0.57 \\
\hline VRO-6 & -0.16 & -0.22 & $0.25 *$ & 0.05 & $0.07 *$ & 0.17 & -0.25 & -3.25 & 0.52 & 0.86 & 0.39 \\
\hline S.E.g ${ }_{i}$ & 0.76 & 1.02 & 0.20 & 0.14 & 0.06 & 0.35 & 3.88 & 7.68 & 0.61 & 1.09 & 2.44 \\
\hline
\end{tabular}

$*$ And $* *$ significant at $5 \%$ and $1 \%$ level of probability, respectively. 
Table.3 Estimates of specific combing ability (sca) effects for different characters in okra

\begin{tabular}{|c|c|c|c|c|c|c|c|c|c|c|c|}
\hline \multicolumn{12}{|c|}{ Traits } \\
\hline Hybrids & $\begin{array}{c}\text { Days to } 50 \\
\% \\
\text { flowering }\end{array}$ & $\begin{array}{c}\text { Days to } \\
\text { first } \\
\text { picking }\end{array}$ & $\begin{array}{c}\text { Fruit } \\
\text { length } \\
\text { (cm) }\end{array}$ & $\begin{array}{l}\text { Fruit } \\
\text { girth } \\
(\mathrm{cm}) \\
\end{array}$ & $\begin{array}{c}\text { Number of } \\
\text { branches } \\
\text { per plant }\end{array}$ & $\begin{array}{l}\text { Internodal } \\
\text { length }(\mathrm{cm})\end{array}$ & $\begin{array}{c}\text { Plant } \\
\text { height } \\
(\mathrm{cm})\end{array}$ & $\begin{array}{c}\text { Fruit } \\
\text { yield per } \\
\text { plant (g) }\end{array}$ & $\begin{array}{l}\text { Number } \\
\text { of fruits } \\
\text { per plant }\end{array}$ & $\begin{array}{c}\text { Number of } \\
\text { seed per } \\
\text { fruit }\end{array}$ & $\begin{array}{c}\text { Days to } \\
\text { last } \\
\text { picking }\end{array}$ \\
\hline $\begin{array}{l}\text { JDNOL-11-1 x } \\
\text { ArkaAnamika }\end{array}$ & -2.23 & 1.94 & 0.01 & $0.73 * *$ & $0.28 * *$ & $1.79 * *$ & $13.82^{*}$ & -11.86 & 0.72 & 0.26 & -4.87 \\
\hline $\begin{array}{l}\text { JDNOL-11-1 x } \\
\text { PusaSawani }\end{array}$ & -1.17 & 1.60 & 0.04 & 0.10 & 0.18 & 0.55 & -3.25 & 22.45 & 0.16 & 2.34 & 1.42 \\
\hline $\begin{array}{l}\text { JDNOL-11-1 x } \\
\text { ParbhaniKranti }\end{array}$ & 0.63 & -1.49 & 0.33 & $1.31 * *$ & 0.15 & -0.86 & 9.82 & 12.08 & -0.32 & 0.73 & 3.45 \\
\hline $\begin{array}{l}\text { JDNOL-11-1 x } \\
\text { GO-2 }\end{array}$ & -2.10 & 1.27 & $0.74 *$ & $-0.48^{*}$ & -0.01 & -0.4 & $-23.13 * *$ & $25.30^{*}$ & -1.18 & -0.68 & 2.65 \\
\hline $\begin{array}{l}\text { JDNOL-11-1 x } \\
\text { VRO-6 }\end{array}$ & 1.30 & 0.76 & 0.006 & 0.02 & 0.18 & -0.74 & -10.30 & $-40.72 * *$ & $-2.84 * *$ & -2.53 & -0.50 \\
\hline $\begin{array}{l}\text { JDNOL-11-3 x } \\
\text { ArkaAnamika }\end{array}$ & $3.04 *$ & -0.84 & $1.01 * *$ & $-0.58 *$ & -0.01 & 0.02 & $14.49^{*}$ & 23.42 & 0.76 & 1.59 & $8.42 *$ \\
\hline $\begin{array}{l}\text { JDNOL-11-3 x } \\
\text { PusaSawani }\end{array}$ & -1.52 & 2.79 & -0.009 & $-0.81 * *$ & -0.05 & -1.08 & -5.19 & 9.34 & 1.43 & -2.85 & -1.64 \\
\hline $\begin{array}{l}\text { JDNOL-11-3 x } \\
\text { ParbhaniKranti }\end{array}$ & -0.72 & 1.16 & $-0.95 * *$ & $0.48^{*}$ & -0.06 & 0.15 & 7.10 & -21.19 & 1.81 & 1.19 & 3.25 \\
\hline $\begin{array}{l}\text { JDNOL-11-3 x } \\
\text { GO-2 }\end{array}$ & 2.02 & -2.26 & $-0.69 *$ & 0.04 & $-0.24 *$ & -0.41 & 1.96 & -11.13 & 0.005 & -1.61 & $-8.57 *$ \\
\hline $\begin{array}{l}\text { JDNOL-11-3 x } \\
\text { VRO-6 }\end{array}$ & 0.74 & $-4.95 * *$ & -0.49 & $-0.82 * *$ & $-0.40 * *$ & 0.96 & -5.33 & -7.70 & -0.56 & 1.53 & -3.61 \\
\hline $\begin{array}{l}\text { JDNOL-11-11 x } \\
\text { ArkaAnamika }\end{array}$ & -0.56 & -2.43 & 0.05 & $0.83 * *$ & -0.01 & -0.39 & 10.46 & $27.91 *$ & 0.05 & -0.5 & 1.67 \\
\hline $\begin{array}{l}\text { JDNOL-11-11 x } \\
\text { PusaSawani }\end{array}$ & 1.20 & -3.21 & -0.37 & $-0.63 * *$ & $-0.22 *$ & -0.36 & $-20.09 * *$ & 1.79 & -0.24 & -1.74 & -6.70 \\
\hline $\begin{array}{l}\text { JDNOL-11-11 x } \\
\text { ParbhaniKranti }\end{array}$ & 0.24 & $3.41 *$ & $-0.65^{*}$ & $-0.85^{* *}$ & -0.06 & 1.01 & 8.36 & $-46.01 * *$ & $2.23 *$ & $-3.69 *$ & -1.50 \\
\hline $\begin{array}{l}\text { JDNOL-11-11 x } \\
\text { GO-2 }\end{array}$ & $2.99 *$ & -0.98 & -0.17 & 0.46 & -0.05 & -0.20 & -8.27 & -17.22 & -0.28 & 0.41 & 0.30 \\
\hline $\begin{array}{l}\text { JDNOL-11-11 x } \\
\text { VRO-6 }\end{array}$ & -0.17 & -1.64 & 0.07 & $0.77 * *$ & 0.12 & $1.22 *$ & $-14.24 *$ & -1.55 & -1.35 & 3.41 & 0.53 \\
\hline
\end{tabular}


Int.J.Curr.Microbiol.App.Sci (2017) 6(9): 1944-1954

\begin{tabular}{|c|c|c|c|c|c|c|c|c|c|c|c|}
\hline $\begin{array}{l}\text { JDNOL-11-12 x } \\
\text { ArkaAnamika }\end{array}$ & -2.00 & $3.69 *$ & 0.26 & -0.18 & 0.04 & 0.08 & $-13.63 *$ & 5.38 & -0.98 & -1.51 & 6.00 \\
\hline $\begin{array}{l}\text { JDNOL-11-12 x } \\
\text { PusaSawani }\end{array}$ & 1.15 & 0.35 & -0.31 & 0.31 & -0.16 & -0.14 & $13.77 *$ & 5.16 & 1.21 & $3.79 *$ & 0.25 \\
\hline $\begin{array}{l}\text { JDNOL-11-12 x } \\
\text { ParbhaniKranti }\end{array}$ & 0.15 & 3.09 & 0.42 & -0.25 & -0.02 & 0.22 & $16.38 * *$ & -3.28 & -1.47 & -1.13 & -0.32 \\
\hline $\begin{array}{l}\text { JDNOL-11-12 x } \\
\text { GO-2 }\end{array}$ & 1.02 & -0.22 & 0.56 & 0.09 & $0.28 * *$ & -0.66 & $12.82^{*}$ & 2.42 & -0.47 & 0.31 & 2.05 \\
\hline $\begin{array}{l}\text { JDNOL-11-12 x } \\
\text { VRO-6 }\end{array}$ & $-4.02 * *$ & -2.05 & 0.13 & $-0.55^{*}$ & 0.10 & -0.77 & -5.55 & $25.39 *$ & 1.31 & 0.64 & -2.29 \\
\hline $\begin{array}{l}\text { JDNOL-11-14 x } \\
\text { ArkaAnamika }\end{array}$ & -1.76 & 1.51 & -0.08 & -0.16 & $-0.24^{*}$ & 0.15 & -9.45 & -6.56 & -0.4 & -1.56 & -0.32 \\
\hline $\begin{array}{l}\text { JDNOL-11-14 x } \\
\text { PusaSawani }\end{array}$ & -0.16 & 1.12 & $0.64 *$ & 0.39 & $0.50 * *$ & 0.93 & $20.00 * *$ & 14.06 & $3.00 * *$ & 3.09 & 5.40 \\
\hline $\begin{array}{l}\text { JDNOL-11-14 x } \\
\text { ParbhaniKranti }\end{array}$ & 0.27 & 0.32 & -0.15 & $-0.80 * *$ & $-0.25^{*}$ & $-2.02 * *$ & -5.55 & 8.91 & -0.58 & 0.97 & -2.68 \\
\hline $\begin{array}{l}\text { JDNOL-11-14 x } \\
\text { GO-2 }\end{array}$ & 0.18 & 1.61 & 0.34 & $0.67 * *$ & $0.22 *$ & 0.69 & -7.76 & -21.95 & 1.84 & -0.68 & -0.86 \\
\hline $\begin{array}{l}\text { JDNOL-11-14 x } \\
\text { VRO-6 }\end{array}$ & 0.90 & 0.47 & -0.30 & 0.11 & $-0.20 *$ & -0.45 & 4.62 & $33.01 * *$ & -0.95 & 1.22 & 0.33 \\
\hline $\begin{array}{l}\text { AOL-07-9 x } \\
\text { ArkaAnamika }\end{array}$ & 1.47 & -1.17 & -0.31 & $-0.77 * *$ & $-0.25^{*}$ & 0.20 & 2.51 & -14.38 & 1.12 & -0.31 & -7.31 \\
\hline $\begin{array}{l}\text { AOL-07-9 x } \\
\text { PusaSawani }\end{array}$ & 1.37 & -0.52 & 0.22 & 0.11 & $0.36^{* *}$ & 1.02 & 0.26 & -12.25 & $-2.69 * *$ & -0.03 & 2.31 \\
\hline $\begin{array}{l}\text { AOL-07-9 x } \\
\text { ParbhaniKranti }\end{array}$ & $-2.43^{*}$ & $-3.61 *$ & -0.42 & -0.02 & 0.005 & 0.11 & -3.62 & 10.50 & $-2.53 *$ & -0.18 & -5.50 \\
\hline $\begin{array}{l}\text { AOL-07-9 x } \\
\text { GO-2 }\end{array}$ & 0.77 & 0.14 & -0.15 & $0.66^{* *}$ & $0.21 *$ & $-1.14^{*}$ & 3.50 & 18.53 & 0.90 & 0.50 & 1.20 \\
\hline $\begin{array}{l}\text { AOL-07-9 x } \\
\text { VRO-6 }\end{array}$ & -0.61 & 0.12 & 0.22 & -0.20 & $-0.35 * *$ & 0.49 & -4.52 & $-29.82 *$ & 0.29 & -3.008 & 7.42 \\
\hline $\begin{array}{l}\text { AOL-08-5 x } \\
\text { ArkaAnamika }\end{array}$ & -0.67 & $-3.25^{*}$ & -0.16 & -0.06 & 0.02 & -0.78 & $16.47 * *$ & 13.002 & -1.01 & 1.24 & 1.04 \\
\hline $\begin{array}{l}\text { AOL-08-5 x } \\
\text { PusaSawani }\end{array}$ & 0.09 & 1.12 & 0.001 & 0.009 & -0.04 & -0.41 & $-14.00 *$ & $-48.62 * *$ & -1.74 & -2.22 & 1.32 \\
\hline $\begin{array}{l}\text { AOL-08-5 x } \\
\text { ParbhaniKranti }\end{array}$ & 0.64 & 0.73 & -0.04 & $-0.81 * *$ & $-0.20 *$ & 0.01 & $-19.35^{* *}$ & -12.07 & $-2.46^{*}$ & -1.77 & -1.39 \\
\hline
\end{tabular}


Int.J.Curr.Microbiol.App.Sci (2017) 6(9): 1944-1954

\begin{tabular}{|c|c|c|c|c|c|c|c|c|c|c|c|}
\hline $\begin{array}{l}\text { AOL-08-5 x } \\
\text { GO-2 }\end{array}$ & -1.31 & 0.55 & $-1.07 * *$ & -0.26 & 0.005 & 0.25 & $25.69 * *$ & -9.70 & $2.97 * *$ & 2.24 & 1.15 \\
\hline $\begin{array}{l}\text { AOL-08-5 x } \\
\text { VRO-6 }\end{array}$ & -1.92 & $-3.71 *$ & 0.09 & $0.51 *$ & $-0.34 * *$ & $1.27 *$ & $12.45^{*}$ & 12.59 & 1.81 & -0.88 & 2.99 \\
\hline $\begin{array}{l}\text { JOL-6k-2 x } \\
\text { ArkaAnamika }\end{array}$ & -0.26 & -2.47 & 0.30 & $0.49 *$ & 0.08 & -0.55 & -12.09 & 3.91 & $-2.84 * *$ & 1.68 & -4.99 \\
\hline $\begin{array}{l}\text { JOL-6k-2 x } \\
\text { PusaSawani }\end{array}$ & -0.96 & 0.92 & 0.37 & $0.69 * *$ & -0.05 & 0.36 & 10.78 & 10.99 & 0.89 & 0.60 & 1.88 \\
\hline $\begin{array}{l}\text { JOL-6k-2 x } \\
\text { ParbhaniKranti }\end{array}$ & $4.07 * *$ & 2.18 & $0.97 * *$ & $-0.56^{*}$ & $0.26^{* *}$ & -0.29 & $-12.51^{*}$ & 20.07 & 0.50 & 1.13 & -0.59 \\
\hline $\begin{array}{l}\text { JOL-6k-2 x } \\
\text { GO-2 }\end{array}$ & $-3.83 * *$ & -0.05 & -0.09 & $-0.53^{*}$ & -0.14 & 0.32 & $-15.15^{*}$ & 10.10 & $2.87 * *$ & -0.62 & 3.39 \\
\hline $\begin{array}{l}\text { JOL-6k-2 x } \\
\text { VRO-6 }\end{array}$ & $4.15^{* *}$ & $3.98 *$ & -0.36 & $0.53 *$ & $0.42^{* *}$ & -0.18 & 7.71 & -0.28 & -0.98 & -1.40 & -4.81 \\
\hline $\begin{array}{l}\text { JOL-08-7 x } \\
\text { ArkaAnamika }\end{array}$ & $5.23 * *$ & 2.23 & 0.17 & $-1.34 * *$ & -0.05 & -0.77 & $-31.30 * *$ & -22.49 & 0.63 & 0.54 & 2.47 \\
\hline $\begin{array}{l}\text { JOL-08-7 x } \\
\text { PusaSawani }\end{array}$ & 0.039 & -0.64 & -0.31 & 0.13 & $-0.40 * *$ & -0.70 & $17.35^{* *}$ & 10.31 & -1.18 & -1.47 & -1.45 \\
\hline $\begin{array}{l}\text { JOL-08-7 x } \\
\text { ParbhaniKranti }\end{array}$ & -1.79 & -2.97 & 0.51 & $1.16^{* *}$ & $0.37 * *$ & $1.86^{* *}$ & 6.70 & $37.08 * *$ & 1.13 & $3.75^{*}$ & 2.13 \\
\hline $\begin{array}{l}\text { JOL-08-7 x } \\
\text { GO-2 }\end{array}$ & 0.23 & -2.45 & 0.17 & -0.39 & -0.15 & -0.34 & $13.47 *$ & 23.58 & $-3.33 * *$ & -1.29 & -3.24 \\
\hline $\begin{array}{l}\text { JOL-08-7 x } \\
\text { VRO-6 }\end{array}$ & -0.10 & $4.12 *$ & 0.13 & $-1.43^{* *}$ & $0.23 *$ & $-1.30^{*}$ & 7.47 & -3.33 & $3.33 * *$ & -1.22 & -3.35 \\
\hline $\begin{array}{l}\text { JOL-08-12 x } \\
\text { ArkaAnamika }\end{array}$ & -2.24 & 0.80 & $-1.27 * *$ & $1.04 * *$ & 0.14 & 0.23 & 8.71 & -18.34 & $1.95^{*}$ & -1.45 & -2.11 \\
\hline $\begin{array}{l}\text { JOL-08-12 x } \\
\text { PusaSawani }\end{array}$ & -0.04 & $-3.55^{*}$ & -0.27 & -0.31 & -0.08 & -0.15 & $-19.63 * *$ & -13.21 & -0.84 & -1.51 & -2.80 \\
\hline $\begin{array}{l}\text { JOL-08-12 x } \\
\text { ParbhaniKranti }\end{array}$ & -1.06 & -2.83 & -0.01 & 0.36 & -0.17 & -0.20 & -7.35 & -6.09 & 1.68 & -1.01 & 3.16 \\
\hline $\begin{array}{l}\text { JOL-08-12 x } \\
\text { GO-2 }\end{array}$ & 0.01 & 2.40 & 0.38 & -0.27 & -0.10 & $1.89 * *$ & -3.13 & -19.92 & $-3.30 * *$ & 1.42 & 1.91 \\
\hline $\begin{array}{l}\text { JOL-08-12 x } \\
\text { VRO-6 }\end{array}$ & -0.27 & 2.90 & 0.51 & $1.05^{* *}$ & $0.22 *$ & -0.50 & 7.70 & 12.42 & -0.05 & 2.23 & 3.29 \\
\hline $\begin{array}{l}\text { S.E.S } \mathbf{s}_{\mathrm{ij}} \\
\end{array}$ & 2.41 & 3.23 & 0.64 & 0.46 & 0.19 & 1.12 & 12.27 & 24.30 & 1.94 & 3.47 & 7.73 \\
\hline
\end{tabular}

* And ** Significant at $5 \%$ and $1 \%$ level of probability, respectively. 
The estimates of specific combining ability (sca) effects of 50 hybrids are presented in table 3. The $s c a$ effects for days to 50 per cent flowering and days to first picking ranged from -4.02 (JDNOL-11-12 x VRO-6) to 5.23 (JOL-08-7 X ArkaAnamika) and -4.95 (JDNOL-11-3 x VRO-6) to 4.12 (JOL-08-7 x VRO-6), respectively. Three hybrids registered significant negative sca effect for days to 50 per cent flowering, while, 5 crosses had significant negative sca effects in case of days to first picking. JDNOL-11-12 x VRO-6 (- 4.02) was the top performing hybrid, while in case of first flowering node JDNOL-11-3 $\mathrm{x}$ VRO-6 (- 4.95) was the best performing hybrid. In the case of fruit length and fruit girth, sca effects varied from -1.27 (JOL-08$12 \mathrm{x}$ ArkaAnamika) to 1.01 (JDNOL-11-3 x ArkaAnamika) and -1.43 (JOL-08-7 x VRO6) to 1.31 (JDNOL-11-1 x ParbhaniKranti), respectively. However, JDNOL-11-3 x ArkaAnamika (1.01) was top performing hybrid for fruit length whereas; JDNOL-11-1 $\mathrm{x}$ ParbhaniKranti (1.31) was top performing hybrid for fruit girth.

The sca effects for number of branches per plant and number of fruits per plant ranged from -0.40 (JDNOL-11-3 x VRO-6) to 0.50 (JDNOL-11-14 x PusaSawani) and from -3.33 (JOL-08-7 x GO-2) to 3.33 (JOL-08-7 $\mathrm{x}$ VRO-6), respectively. Eleven and six hybrids recorded highest significant positive sca effects, respectively. JDNOL-11-14 x PusaSawani (0.50) and JOL-08-7 x VRO-6 (3.33) were the top performing hybrids for the traits number of branches per plant and number of fruits per plant, respectively.

For fruit yield per plant and days to last picking, sca effects ranged from -48.62 (AOL-08-5 x PusaSawani) to 37.08 (JOL-087 x ParbhaniKranti) and -8.57 (JDNOL-11-3 $\mathrm{x}$ GO-2) to 8.42 (JDNOL-11-3 $\mathrm{x}$ ArkaAnamika), respectively, while, the numbers of hybrids revealing good (significant positive) specific combining effects were five and one for both the traits, respectively. JOL-08-7 x ParbhaniKranti (37.08) and JDNOL-11-3 x ArkaAnamika (8.42) was the best performing hybrids for the traits fruit yield per plant and days to last picking, respectively. The sca effects for stem internodal length ranged from -2.02 (JDNOL11-14 x ParbhaniKranti) to 1.89 (JOL-08-12 x GO-2). Five hybrids exhibit significant positive gca effect. Whereas, JOL-08-12 x GO-2 (1.89) was the top promising hybrid recorded significant positive gca effect. Ten and one crosses recorded significant negative sca effects in plant height and number of seeds per fruit, respectively. For this trait sca effects varied from -31.30 (JOL-08-7 x ArkaAnamika) to 25.69 (AOL-08-5 x GO-2) and -3.69 (JDNOL-11-11 x ParbhaniKranti) to 3.79 (JDNOL-11-12 x PusaSawani). JOL08-7 x ArkaAnamika (-31.30) and JDNOL11-11 x ParbhaniKranti (-3.69) were the top performing hybrids for this trait, respectively.

It can be seen that the crosses exhibiting desirable and significant sca effects for fruit yield per plant and total fruit yield were also associated with high and favourablesca effects for multiple yields contributing traits. The highest fruit yielding hybrid JOL-08-7 x ParbhaniKranti (Good x Good) had significant desirable sca effect for fruit yield and some of the yield components, followed by a cross, JDNOL-11-12 x VRO-6. Corresponding to these findings, Das et al., (2013) reported that positive sca effects were discernible in the hybrids involving both the parents possessing significant positive gca effects. These good $\mathrm{x}$ good combinations could result in the capitalization of nonadditive (Dominance $\mathrm{x}$ dominance variance) effects over the super structure of the additive gene effects. Hybrids involving both the parents possessing significant positive gca effects (good x good) with higher significant sca effects for number of fruits per plant and 
fruit yield per plant in okra have been earlier reported by Raghuvanshietal., (2011), Wammandaet al., (2010), Singh, (2011), Aulakhet al., (2012), Medagamet al., (2012), Das etal., (2013), Atotkaret al., (2014) and Katagiet al., (2015).

The $g c a$ effects of the parents indicated that parent JOL-08-7 was good general combiner for fruit yield and other yield attributing traits. SCA effects of the hybrid revealed that hybrid JOL-08-7 x ParbhaniKranti (Good x Good) was found to be the best combination for fruit yield per plant and other yield attributing traits like a number of branches, internodal length and fruit girth.

\section{Acknowledgement}

The author is thankful to the Seed Spices Research Station, S.D. Agriculture University, Jagudan, Gujarat, India for technical and moral support.

\section{References}

Akotkar, P. K., De, D. K. and Dubey, U. 2014. Genetic studies on fruit yield and yield attributes of okra (Abelmoschus esculentus (L.) Moench.). Elect. J. Pl.Breed., 5(1): 38 - 44.

Allard, R.W., 1960. Principles of Plant Breeding, John Willey and Sons.Inc. London.

Anonymous, 2015. Indian Horticulture Database, National horticulture Board. www. nhb.gov.in.

Aulakh, P. S., Dhall, R. K. and Singh, J. 2012. Genetics of early and total yield in okra (Abelmoschus esculentus (L.) Moench). Veg. Sci., 39(2): 165 - 168.

Das, S., Chattopadhyay, A., Dutta, S., Chattopadhyay, S. and Hazra, P. 2013. Breeding okra for higher productivity and yellow vein mosaic tolerance. I. $J$. of Veg. Sci., 19: 58 - 77.
FAOSTAT, 2013. Production- Crops (2013) data.Food and Agriculture Organization of the United Nations. http://www.fao.org/faostat

Jaiprakashnarayan, R.P., Prashanth, S.J., Mulge, R. and Madalageri, M.B. 2008. Study on heterosis and combining ability for earliness and yield parameters in okra (Abelmoschus esculentus (L.) Moench). The Asian J. of Horti, 3 (1): 136 - 141.

Jethava, B.A., 2014. Heterosis and combining ability in Okra (Abelmoschus esculentus (L.) Moench). M.Sc. Thesis, Submitted to Navsari Agricultural University, Navsari.

Katagi, A., Tirakannanvar, S. and Jagadeesha, R. C. 2015.Combining ability through diallel analysis in okra (Abelmoschus esculentus (L.) Moench). Green FarmingInt. J., 6(1): 26 - 29.

Kempthrone, O., 1957.An Introduction to Genetic Statistics.John Wiley and Sons.Inc. Newyork. 545.

Khatik, K. R., Chaudhary, R. and Khatik, C. L. 2012.Heterosis studies in okra. Annals of Horti, 5(2): 213 - 218.

Kumar, N., 2006.Breeding of Horticultural crops. New Delhi: New India Publishing Agency, pp 173-7.

Medagam, T. R., Kadiyala, H., Mutyala, G. and Hameedunnisa Begum. 2012. Heterosis for yield and yield components in okra. Chilean J. Agric. Res., 72(3): 316 - 325.

Obiadalla Ali., Eldekashi, M. H. and Helaly, A. A., 2013.Combining ability and heterosis studies for yield and its components in some cultivars of okra.American-Eurasian J. Agric. \& Environ. Sci., 13(2): 162 - 167.

Raghuvanshi, M., Singh, T. B., Singh, A. P., Singh, U., Singh, P. and Singh, B. 2011.Combining ability in okra.Veg.Sci, 38(1): 26 - 29.

Singh, D., Sharma, B. R. and Singh, H. C. 
1991.Genetical analysis to predict the breeding potential in an intervarietal cross in okra (Abelmoschus esculentus (L.) Moench) for improvement in fruit and seed yield. In "Golden Jubilee Symposium on Genetic Research and Education: Current Trends and next Fifty years." Abstr, 2: 605.

Singh, D.R., Singh, P.K., Syamal, M.M. and Gautam, S.S. (2009).Studies on combining ability in okra. Indian J.Horti, 66 (2): 277 - 280.

Singh, S. P., 2011. Combining ability analysis for yield and yield contributing characters in okra. Veg. Sci., 38(2): 212 $-214$.

Singh, S., Singh, B. and Pal, A.K. 2006.Line $\mathrm{x}$ Tester analysis of combining ability in
okra.Indian J. Hort., 63(4): 397 - 401.

Sood, S., and Kalia, P. 2001. Heterosis and combining ability studies for some quantitative traits in okra (Abelmoschus esculentus (L.) Moench). Haryana J. Hort. Sci., 30(1 \& 2): 92 - 94.

Wakode, M. M., Bhave, S. G., Navhale, V. C., Dalvi, V. V., Devmore, J. P. and Mahadik, S. G. 2016. Combining ability studies in okra (Abelmoschus esculentus L. Moench). Elect. J. Pl.Breed., 7(4): 1007-1013.

Wammanda, D. T., Kadams, A. M. and Jonah, P. M. 2010. Combining ability analysis and heterosis in a diallel cross of okra. African J. of Agri. Res., 5(16): 2108 2115.

\section{How to cite this article:}

Satish, K., A.V. Agalodiya and Prajapati, D.B. 2017. Combining Ability for Yield and Its Attributing Traits in Okra [Abelmoschus esculentus (L.) Moench]. Int.J.Curr.Microbiol.App.Sci. 6(9): 1944-1954. doi: https://doi.org/10.20546/ijcmas.2017.609.238 